\title{
Movements Involuntary, CTCAE
}

National Cancer Institute

\section{Source}

National Cancer Institute. Movements Involuntary, CT CAE. NCI Thesaurus. Code C143677.

A disorder characterized by uncontrolled and purposeless movements. 